\title{
Clinical-Radiological, Pathological Profile and Treatment Outcome of Patients with Haemoptysis
}

Tinku Joseph*, Sreeraj Nair and James PT

Department of Pulmonary Medicine, Amrita Institute of Medical Sciences, Kochi, Kerala, India

\begin{abstract}
Background: Haemoptysis is a frequently occurring life threatening situation which can occur in various pulmonary conditions. Managing a case of haemoptysis is often difficult due to a large spectrum of causative factors. The effective control of haemoptysis depends upon identification of the etiology and localization of the site of bleeding
\end{abstract}

Method: This was a two year cross sectional study of 102 patients who presented with active haemoptysis. Data from these patients was collected for the analyzing the underlying cause, diagnostic modality, treatment approach used and outcome of it in patients suffering from life-threatening haemoptysis.

Results: Old Pulmonary tuberculosis, (34.31\%), bronchiectasis $(25.49 \%)$, lung cancer $(11.76 \%)$, aspergilloma $(10.78 \%)$ accounted for the most common etiologies of haemoptysis. 12 patients $(11.76 \%)$, out of 102 had mild haemoptysis, 26 patients $(25.49 \%)$ had moderate haemoptysis and 64 patients $(62.74 \%)$ had massive/severe haemoptysis. CT chest was the most sensitive diagnostic test when employed alone, with positive yield of $93.14 \%$. Among study population 61 patients $(59.80 \%)$ were treated conservatively, 39 patients $(38.23 \%)$ were treated by bronchial artery embolisation (BAE) and 2 patients underwent surgery for management of recurrent haemoptysis. Most of the patients were treated conservatively, but in case of significant haemoptysis, Bronchial artery embolization was performed than the conservative treatment. Success rate of conservative treatment group on a long term basis was $72.13 \%$, BAE group $89.47 \%$, surgical treatment group was $100 \%$, without any significant statistical significance ( $p$ value $=0.090$ ). The duration of hospitalization among conservative treatment group was 4.37 days and BAE group was 10.51 days ( $P$-value $<0.001)$.

Conclusion: The most common causes of haemoptysis were old pulmonary tuberculosis and bronchiectasis Emergency bronchial artery embolisation is a better option than medical treatment for immediate cessation of haemoptysis. The successful treatment of haemoptysis requires thorough diagnostic evaluation and close interdisciplinary collaboration among pulmonologists, radiologists, and thoracic surgeons.

Keywords: Haemoptysis; Tuberculosis; Lung cancer; Bronchiectasis; Bronchial artery embolisation

\section{Introduction}

Hemoptysis is one of the commonest manifestations of various pulmonary diseases encountered by a pulmonologist. A major problem in managing hemoptysis is the wider spectrum of etiological factors that may result in hemoptysis. The effective management of hemoptysis depends upon identification of the etiology and localization of the site of bleeding [1]. Haemoptysis is defined as the expectoration of blood derived from the lungs as a result of pulmonary or bronchial vascular haemorrhage [2]. It remains the critical problem for both patient as well as treating physician.

Massive haemoptysis is an alarming and life-threatening illness associated with a mortality of up to $78 \%$, which requires immediate medical attention. Earlier studies have demonstrated an improved outcome in patients who were treated with surgical resection of the affected lobe of the lung compared with medical treatment alone [2]. Over the past decade, bronchial artery embolisation has been considered as the treatment of choice, especially in patients who are not surgical candidates, with success rates of up to $90 \%$.

Earlier studies have documented the aetiology of massive haemoptysis to be mostly due to tuberculosis and post-tuberculous bronchiectasis [2]. More recent reviews have documented a change in this pattern with lung cancer and bronchiectasis as the predominant cause of massive haemoptysis in Western countries.

The aim of our study was to evaluate the etiology, diagnostic and treatment modalities used and its outcome in patients with lifethreatening haemoptysis.

\section{Materials and Methods}

This cross sectional study was carried out in Pulmonary Medicine Department and allied clinics of Amrita institute of medical sciences, Kochi. Total 102 adult patients presented with haemoptysis were enrolled during the period from February 2014 to August 2016.

\section{Patient selection}

Inclusion criteria:

- All patients presenting with active haemoptysis to Pulmonary Medicine department and other allied clinics.

Exclusion criteria:

- Patients less than 18 years of age

- Known coagulopathies

\section{Procedure}

Detailed case history and a thorough clinical examination were done

${ }^{*}$ Corresponding author: Dr. Tinku Joseph, MD, DM, FCCP., Consultant Interventional Pulmonologist, Department of Pulmonary Medicine, Amrita Institute of Medical Sciences, Kochi, Kerala, India, Tel: ;0484-2858660 E-mail: tinkujoseph2010@gmail.com

Received November 27, 2017; Accepted December 18, 2017; Published December 26, 2017

Citation: Joseph T, Nair S, James PT (2017) Clinical-Radiological, Pathological Profile and Treatment Outcome of Patients with Haemoptysis. J Pulm Respir Med 7: 437. doi: 10.4172/2161-105X.1000437

Copyright: (C) 2017 Joseph T, et al. This is an open-access article distributed under the terms of the Creative Commons Attribution License, which permits unrestricted use, distribution, and reproduction in any medium, provided the original author and source are credited. 
among all patients in our study population. Also we collected details on previous history of haemoptysis, previous lung disorders and smoking status of the patient. Special emphasis was made to grade quantity and frequency of the haemoptysis. Chest X-ray abnormalities, hemoglobin level and the presence of respiratory failure were documented. Results of other investigations such as computed tomography (CT) chest, fibreoptic bronchoscopy (FOB) and bronchial angiography were also noted. Patients in our study population were followed up for 6 months. The efficacy of various diagnostic and treatment modalities and recurrence of hemoptysis were also noted.

\section{Statistical analysis}

Statistical analysis was performed using IBM SPSS version 20.0 software. Categorical variables were presented using frequency and percentage. Numerical variables were expressed using mean and standard deviation. Descriptive statistics were also used.

\section{Results}

\section{Demographics}

In our study, a total number of 102 patients admitted with features of active haemoptysis were enrolled in the baseline group after proper screening and ensuring that they satisfy the inclusion criteria. Written consent was taken from all patients. 32 (31.4\%) females and 70 $(68.6 \%)$ male patients constituted our study population (Figure 1 ). The maximum numbers of patients (40) were between 51-60 years of age group. This was followed by 31 patients in age groups between 41-50 years of age.

\section{Clinical characteristics}

Among the total 102 patients enrolled in the study population, $54(52.94 \%)$ patients were active smokers, $10(9.80 \%)$ patients were reformed smokers and 38 (37.26\%) patients were non-smokers. 36 patients had systemic hypertension, 15 patients had type 2 diabetes, 16 patients were suffering from COPD and 35 patients had the history of old pulmonary TB. 12 cases out of 102 cases were mild (11.76\%), 26 cases moderate $(25.49 \%)$, and 64 cases massive/severe hemoptysis $(62.74 \%)$.

\section{The causes of hemoptysis}

Old pulmonary Tuberculosis (old PTB) was the commonest cause of haemoptysis among the study population (Table 1). It comprised 35 patients $(34.31 \%)$. Second common cause of haemoptysis noted in our study was bronchiectasis (26 patients, 25.49\%). Other notable causes of

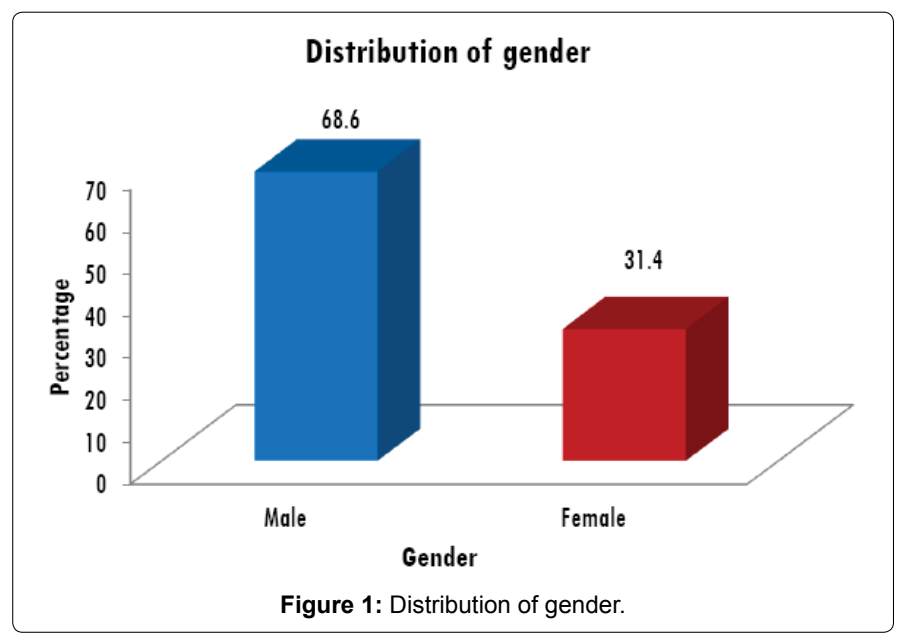

haemoptysis seen in our study were 12 cases of lung cancer (11.76\%), 11 cases of aspergilloma (10.78\%), 4 cases of pneumonia (3.92\%), 1 patient each of lung abscess $(0.98 \%)$ and on oral anticoagulants (0.98\%). Also 8 cases of haemoptysis due to unknown/cryptogenic causes (7.84 \%) were also there in our study population.

Among 64 massive haemoptysis patients in our study population, 30 patients had history pulmonary Tuberculosis (46.87\%), the highest frequency, followed by 17 cases of bronchiectasis (26.56\%), 6 cases of lung cancer (9.37\%), aspergilloma (7.81\%), 3 cases of active pulmonary TB (4.68\%), 1 case of massive hemoptysis was reported due to pneumonia, patient on anti coagulants and due to unknown cause (Table 1).

\section{Modality of diagnosis}

CT scan chest was performed for all 102 patients, of which pulmonary lesions were detected in 93 patients (91.18\%) (Table 2). Lung parenchymal cavitatory lesions were seen in 36 patients (35.29\%). Findings of bronchiectasis were noted in CT scan of 26 patients (25.54\%). Aspergilloma (cavitating lesion with fungal ball) was seen in 11 cases $(10.78 \%)$. Lung mass was seen in 12 cases $(11.86 \%)$. Out of the total 102 patients with haemoptysis who underwent CT chest, in 9 patients there was no obvious radiological abnormality noted. Fiberoptic bronchoscopy was done for 42 patients (41.17\%) in order to localize the source of bleeding and for obtaining BAL for AFB smear, Gene Xpert, BACTEC culture, routine bacterial culture and cytology. It was also done for obtaining tissue diagnosis in patients with high suspicion of lung cancer on the basis of CT chest findings. Active endobronchial bleed was noted in 10 patients who underwent fiberoptic bronchoscopy and endobronchial mass lesion was visualized in 8 patients as well.

All 26 cases of bronchiectasis were diagnosed by CT chest. Fiberoptic bronchoscopy was performed for 4 cases (1.04\%), of which 2 cases $(50 \%)$ showed abnormalities. Bronchial angiography was performed for 24 cases (92.30\%), of which in 20 cases (83.33\%) bleeding site was detected. CT chest was done for all 35 patients with history of old pulmonary tuberculosis who presented with haemoptysis. Findings were confirmed in all the 35 cases (100\%). Fiberopticbronchoscopic evaluation was done for 8 patients 22.85\%) in order to rule out endobronchial bleed. In 2 cases (25\%) active bleed was noted. 20 cases (57.14\%) underwent bronchial angiography to localize the source of bleed and this was followed by bronchial artery embolisation in 14 cases (70\%).

\section{Treatment outcome $v s$. quantity of haemoptysis}

A total of 61 cases $(59.80 \%)$ were managed conservatively with medicines (Intravenous/oral Ethamsylate, Tranexamic acid, cough suppressants). Bronchial artery embolisation (BAE) was done for 39 patients $(38.23 \%)$ for attaining haemostasis. 2 patients $(1.96 \%)$

\begin{tabular}{|l|c|c|}
\hline \multicolumn{1}{|c|}{ Causes } & No: of patients & Percentage (\%) \\
\hline Bronchiectasis & 26 & 25.49 \\
\hline Old PTB & 35 & 34.31 \\
\hline Active PTB & 4 & 3.92 \\
\hline Carcinoma Lung & 12 & 11.76 \\
\hline Aspergilloma & 11 & 10.78 \\
\hline Lung Abscess & 1 & 0.98 \\
\hline Pneumonia & 4 & 3.92 \\
\hline On anticoagulants & 1 & 0.98 \\
\hline Unknown cause/Cryptogenic & 8 & 7.84 \\
\hline
\end{tabular}

Table 1: Etiological distribution of patients with haemoptysis. 
Citation: Joseph T, Nair S, James PT (2017) Clinical-Radiological, Pathological Profile and Treatment Outcome of Patients with Haemoptysis. J Pulm Respir Med 7: 437. doi: 10.4172/2161-105X.1000437

Page 3 of 5

\begin{tabular}{|c|c|c|c|c|c|c|}
\hline Causes & $\begin{array}{l}\text { CT chest } \\
\text { performed }\end{array}$ & $\begin{array}{c}\text { CT chest } \\
\text { positive result }\end{array}$ & FOB performed & $\begin{array}{l}\text { FOB positive } \\
\text { result }\end{array}$ & $\begin{array}{c}\text { Bronchial angiography } \\
\text { performed }\end{array}$ & $\begin{array}{c}\text { Bronchial angiography } \\
\text { positive result }\end{array}$ \\
\hline Bronchiectasis & $26(100)$ & $26(100)$ & $4(1.04)$ & $2(50)$ & $24(92.30)$ & $20(83.33)$ \\
\hline Old PTB & $35(100)$ & $35(100)$ & $8(22.85)$ & $2(25)$ & $20(57.14)$ & $14(70)$ \\
\hline Active PTB & $4(100)$ & $4(100)$ & $2(50)$ & $2(100)$ & $0(0)$ & $0(0)$ \\
\hline Carcinoma Lung & $12(100)$ & $12(100)$ & $12(100)$ & $10(83.33)$ & $2(16.66)$ & $1(50)$ \\
\hline Aspergilloma & $11(100)$ & $11(100)$ & $5(45.45)$ & $0(0)$ & $6(54.54)$ & $4(66.66)$ \\
\hline Lung abscess & $1(100)$ & $1(100)$ & $0(0)$ & $0(0)$ & $0(0)$ & $0(0)$ \\
\hline Pneumonia & $4(100)$ & $4(100)$ & $2(50)$ & $2(100)$ & $0(0)$ & $0(0)$ \\
\hline On anticoagulants & $1(100)$ & $0(0)$ & $1(100)$ & $0(0)$ & $0(0)$ & $0(0)$ \\
\hline Unknown cause/Cryptogenic & $8(100)$ & $2(25)$ & $8(100)$ & $0(0)$ & $8(0)$ & $0(0)$ \\
\hline Total & 102 & 95 (93.14) & $42(44.52)$ & $22(52.38)$ & $60(58.82)$ & $39(65)$ \\
\hline
\end{tabular}

Table 2: The methodology of diagnosis.

\begin{tabular}{|c|c|c|c|c|c|c|}
\hline Treatment outcome & Short term Success & Short term recurrence & P-value & Long term success & Long term recurrence & P-value \\
\hline Conservative Treatment & $41(67.21)$ & $20(32.78)$ & \multirow{3}{*}{0.180} & $44(72.13)$ & $17(27.86 \%)$ & \multirow{3}{*}{0.090} \\
\hline BAE & $32(82.05)$ & $7(17.94)$ & & $34(89.47)$ & $4(10.52 \%)$ & \\
\hline Surgery & $2(100)$ & 0 & & $2(100)$ & 0 & \\
\hline
\end{tabular}

Table 3: Treatment outcome according to the treatment method used. Values are presented as number (\%).

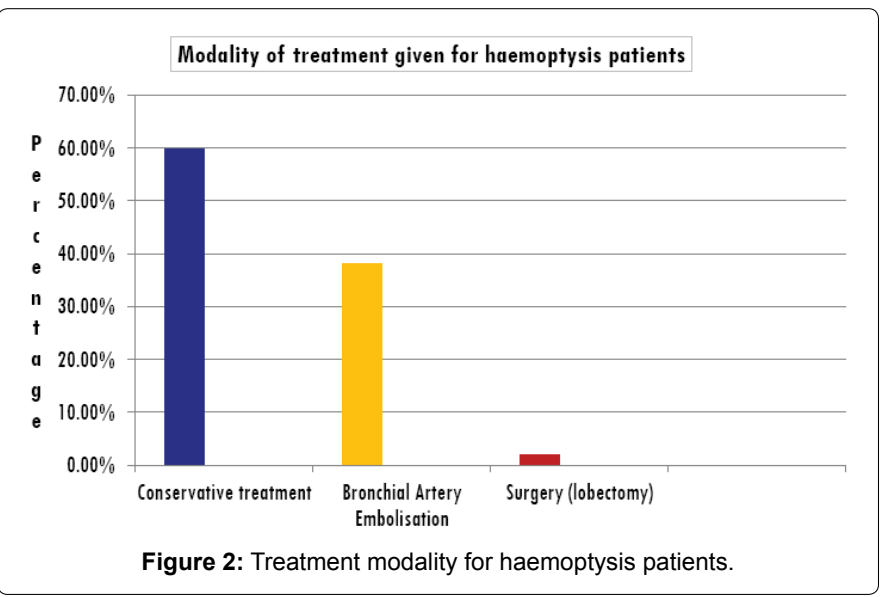

Duration of hospitalization according to the treatment method

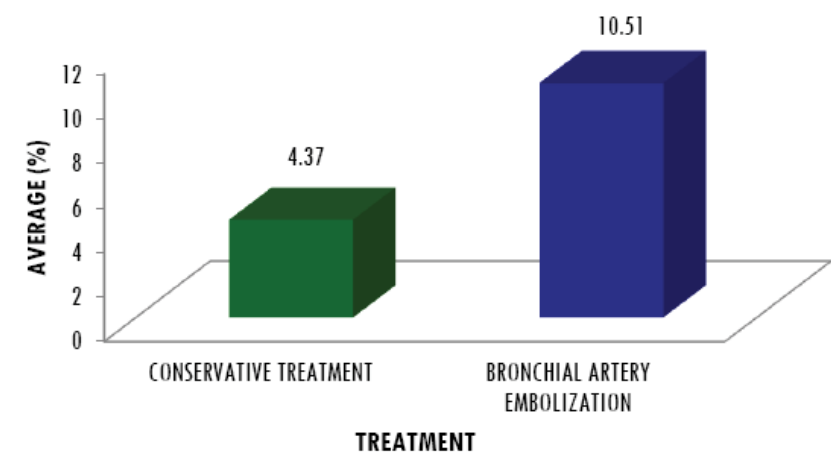

Figure 3: Duration of hospitalization according to the treatment method.

underwent surgery (loebectomy) for control of haemoptysis (Figure 2). Among mild hemoptysis, 7 cases $(58.33 \%$ ) out of 12 cases were controlled during first 3 days, while 16 cases (61.53\%) out of 26 cases were controlled in moderate haemoptysis. Among cases of massive hemoptysis, 51 cases $(80.95 \%)$ out of 64 cases were controlled.
However p-value obtained (short term recurrence 0.078 and long term recurrence 0.563 ) was not statistically significant when recurrence rate of haemoptysis was compared among all the three grades.

\section{Outcome of the treatment versus method used to for attaining haemostatis}

The short-term and long-term control of haemoptysis, in-hospital mortality rates were investigated according to treatment method. In the short-term control, the success rate of conservative treatment group was $67.21 \%$, BAE group $82.05 \%$, surgical treatment group $100 \%$, showing that the success rate of surgery in controlling haemoptysis was high. However the number of cases enrolled in surgical group was very minimal and success rate of it could not be compared with conservative treatment and BAE to assess statistical significance. When success rate of the long-term control was taken into account, the conservative treatment group was $72.13 \%$, BAE group $89.47 \%$, surgical treatment group $100 \%$, without any significant difference $(\mathrm{p}=0.090)$ (Table 3$)$.

\section{Duration of hospitalization according to treatment method} used

A comparison was made for the duration of hospitalization for each treatment group. The hospitalization duration of conservative treatment was 4.37 days; BAE group was 10.51 days, which indicates that duration of hospitalization for conservative treatment was significantly shorter than other treatment groups ( $\mathrm{P}$-value $<0.001)$ (Figure 3 ).

\section{Discussion}

Hemoptysis is one of the most common symptom in patients with various pulmonary diseases and often a life threatening condition. Massive hemoptysis occurs in approximately $1.5 \%$ of patients presenting with haemoptysis. The prognosis is poor, and the mortality by bleeding or asphyxia reaches $50-60 \%[3,4]$. Etiological pattern of haemoptysis has changed in the developed countries, with pulmonary tuberculosis is becoming less important as a cause [5]. However in developing countries, pulmonary tuberculosis is still to be the major cause of haemoptysis [6].

A quite significant number of patients have inactive tuberculosis as evident by the present study where 35 patients (34.31\%). Second common cause of haemoptysis noted in our study was bronchiectasis (26 patients, $25.49 \%$ ). In another recent study, bronchiectasis was found 
to be most common cause of hemoptysis in $34.55 \%$ of patients. Abal et al. [1] also found bronchiectasis as most common cause in $20 \%$ of patients. Hirschberg et al. [7] also found same findings. MacGuinness et al. [8] recorded hemoptysis in $25 \%$ bronchiectasis and $16 \%$ tuberculosis cases. Other notable causes of haemoptysis seen in our study were 12 cases of lung cancer (11.76\%), 11 cases of aspergilloma (10.78\%), 4 cases of pneumonia (3.92\%), 1 patient each of lung abscess $(0.98 \%)$ and on oral anticoagulants $(0.98 \%)$.

In our study haemoptysis was more commonly found in male patients 70 cases $(68.60 \%)$ and female patients constituted 32 cases (31.40\%). Hemoptysis was commonly found in the age group 51-60 years ( 40 cases), followed by $41-50$ years age group ( 31 cases). The mean age of our study population was 48.81 . Male female ratio in our study was found to be 2.18:1, concluding males are twice more susceptible to develop hemoptysis than females. Majority of the patients (62.74\%) in the present study presented with severe haemoptysis, $25.49 \%$ with moderate haemoptysis and $11.76 \%$ of patients presented with mild haemoptysis.

In our study chest CT was performed for all patients (102 cases), of which pulmonary lesions were detected in 93 cases (91.18\%). Lung parenchymal cavitatory lesions were seen in 36 patients $(35.29 \%)$. Findings of bronchiectasis were noted in CT scan of 26 patients (25.54\%). Aspergilloma (cavitating lesion with fungal ball) was seen in 11 cases $(10.78 \%)$. Lung mass was seen in 12 cases $(11.86 \%)$. Out of the total 102 patients with haemoptysis who underwent CT chest, in 9 patients there was no obvious radiological abnormality noted. All 26 cases of bronchiectasis were diagnosed by chest CT.

Chest CT has been shown to be accurate in the diagnosis of a wide range of bronchial abnormalities [9]. Bronchoscopy is a reliable method in locating the site of bleeding, removal of obstructive clots, direct visualization of endobronchial tumors, foreign bodies, granulomas, and infiltrations. It also allows collection of histologic samples, but is not useful in detecting peripheral tumors [10]. Bronchial angiography can localize potential bleeding sites and treat the bleeding by embolisation [11]. The combined use of bronchoscopy and CT chest has the best yield in evaluating haemoptysis [12].

In our study 61 cases $(59.80 \%)$ were managed conservatively with medicines (Intravenous/oral Ethamsylate, Tranexamic acid, cough suppressants). Bronchial artery embolisation (BAE) was done for 39 patients $(38.23 \%)$ for attaining haemostasis. 2 patients $(1.96 \%)$ underwent surgery (lobectomy) for control of haemoptysis. During follow up period the short-term, long-term control of haemoptysis and in-hospital mortality rates were assessed according to treatment method. In the short-term control, the success rate of conservative treatment group was $67.21 \%$, BAE group $82.05 \%$, surgical treatment group $100 \%$, showing that the success rate of surgery in controlling haemoptysis was high. However the number of cases enrolled in surgical group was very minimal and success rate of it could not be compared with conservative treatment and BAE to assess statistical significance [13].

Since Bronchial artery embolisation (BAE) was first reported by Rémy et al. [9], it has been widely used because it can stabilize the condition of patients, gaining time for the preparation of surgery for acute hemoptysis, along with temporary treatment. The early success rate of BAE is $73-98 \%$. In a Korean study, the early success rate is $95 \%$, and long-term success rate is $36 \%$ [8]. In our study, the first-line success rate of BAE was $82.05 \%$, while the long-term success rate was $89.47 \%$, without statistical difference. Long term recurrence of haemoptysis was seen in $10.52 \%$ of cases. Recurrence might be due to incomplete embolisation, revascularization, or recanalization. In several reports, the major conditions in which recurrence has been noted include tuberculosis, aspergilloma and lung cancer [14]. Definitive treatment of the underlying condition is essential to provide cure of the disease. In cases of recurrent bleed, repeat BAE has proven useful.

Bronchial artery embolisation is relatively a safe procedure. In our study Bronchial artery embolisation (BAE) was very well tolerated procedure for most of the patients (32 cases). However one patient developed paraplegia due to occlusion of the aberrant spinal artery by dislodged gel foam particles after BAE. In the comparison of hospitalization duration by group, conservative treatment group was the shortest among other groups. The hospitalization duration of conservative treatment was 4.37 days; BAE group was 10.51 days, indicating that the hospitalization duration of conservative treatment was significantly shorter than other treatment groups ( $\mathrm{P}$-value $<0.001$ ). Even though length of hospital stays was more after surgery, this group of cases was omitted from analysis due to less number of cases [15]. This is an expected result in that the patients who are clinically stabilized and have a small amount of hemoptysis would first try conservative treatment. However, the short-term success rate was higher in the BAE group or surgical treatment group than conservative treatment group. In a similar type of study Ram lee et al. [12] compared the duration of hospitalization for each treatment group. The hospitalization duration of conservative treatment was $7.4 \pm 7.0$ days BAE group $14.9 \pm 15.8$ days, surgical treatment group $20.9 \pm 16.3$ days, indicating that the hospitalization duration of conservative treatment was significantly shorter than other treatment groups $(\mathrm{p}=0.005)$.

\section{Limitations}

One possible drawback of our study could be that some of the patients lost to follow up for a longer period which might affect the results. Nevertheless, our study reports one of the largest series of medical inpatients and may provide a useful framework for the therapeutic management of haemoptysis in this clinical setting.

\section{Conclusion}

The most common causes of haemoptysis were old pulmonary tuberculosis and bronchiectasis. Emergency bronchial artery embolisation is a better option than medical treatment for immediate cessation of haemoptysis. However, patients exhibiting massive bleeding or those with malignancy had a poorer prognosis. The successful treatment of haemoptysis requires thorough diagnostic evaluation and close interdisciplinary collaboration among pulmonologists, radiologists, and thoracic surgeons.

\section{References}

1. Abal AT, Nair PC, Cherian J (2001) Haemoptysis: Aetiology, evaluation and outcome a prospective study in a third-world country. Respir Med 95: 548-552.

2. Firth JR (1990) Kendig's disorders of the respiratory tract in children. ( $5^{\text {th }}$ edn.), WB Saunders Company, Philadelphia.

3. Uflacker R, Kaemmerer A, Neves C, Picon PD (1983) Management of massive hemoptysis by bronchial artery embolization. Radiology 146: 627-634.

4. Remy J, Voisin C, Dupuis C, Beguery P, Tonnel AB, et al. (1974) Treatment of hemoptysis by embolisation of the systemic circulation. Ann Radiol (Paris) 17: 5-16.

5. Bidwell JL, Pachner RW (2005) Hemoptysis: Diagnosis and management AmFam Physician 72: 1253.

6. Ashraf $\mathrm{O}$ (2006) Hemoptysis: A developing world perspective. BMC Pulmonary Med 6: 1.

7. Hirshberg B, Biran I, Glazer M, Mordechai R (1997) Haemoptysis: Aetiology evaluation and outcome in a tertiary referral Hospital. Chest 112: 440-444.

8. McGuinness G, Beacher JR, Harkin TJ (1994) Haemoptysis: Prospective high 
Citation: Joseph T, Nair S, James PT (2017) Clinical-Radiological, Pathological Profile and Treatment Outcome of Patients with Haemoptysis. J Pulm Respir Med 7: 437. doi: 10.4172/2161-105X.1000437

resolution CT/bronchoscopic correlation. Chest 105: 1155-1162.

9. Rémy J, Arnaud A, Fardou H, Giraud R, Voisin C (1977) Treatment of hemoptysis by embolization of bronchial arteries. Radiology 122: 33-37.

10. Santiago S, Tobias J, Williams AJ (1991) A reappraisal of the causes of hemoptysis. Arch Intern Med 151: 2449.

11. Brinson GM, Noone PG, Mauro MA, Knowles MR, Yankaskas JR, et al. (1998) Bronchial artery embolisation for the treatment of hemoptysis in patients with cystic fibrosis. Am J RespirCrit Care Med 157: 1951-1958.

12. Lee BR, Yu JY, Ban HJ, Oh IJ, Kim KS (2012) Analysis of patients with hemoptysis in a tertiary referral hospital. Tuberc Respir Dis (Seoul) 73: 107-114.

13. Hatipoglu ON, Osma E, Manisali M (1996) High resolution computed tomographic findings in pulmonary tuberculosis. Thorax 51: 397-402.

14. Pragati Rao D, Niranjan Reddy $\mathrm{CH}$, Padmaja A (2015) Aetiology of haemoptysis: Study in a medical college. Int J Current Med Appl Sci 8: 24-26.
15. Boren HG, Busey J, Corpe RF, Grzybowski S, Newman MM (1966) The management of haemoptysis. Am Rev Respir Dis 93: 471. 